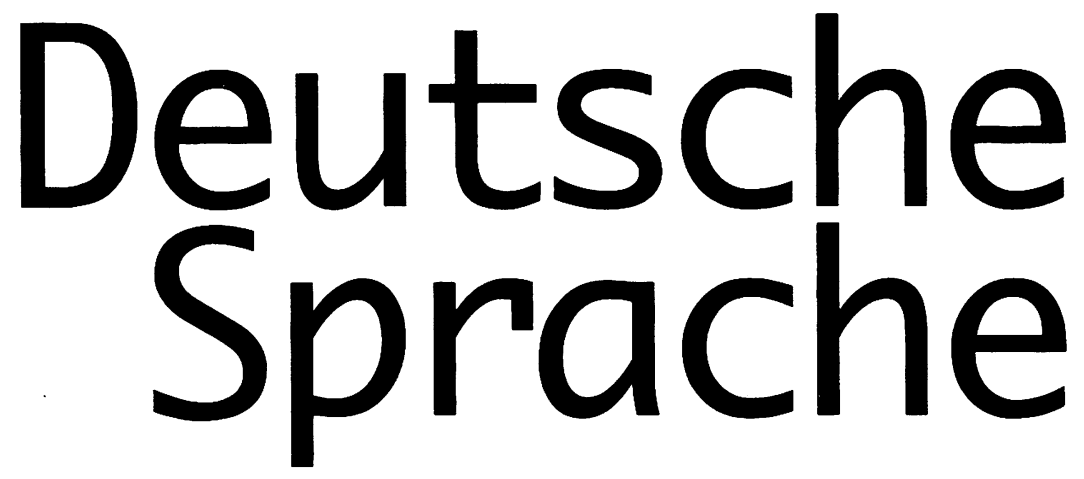

ZEITSCHRIFT

F Ü R

THEORIE

PRAXIS

37. Jahrgang 2009

DOKUMENTATION

Im Auftrag des

Instituts für deutsche Sprache, Mannheim

herausgegeben von

Ludwig M. Eichinger (Geschäftsführung), Martine Dalmas 


\section{JAHRESINHALTSVERZEICHNIS 2009}

Seite

1 Ludwig M. Eichinger (Mannheim)/Martine Dalmas (Paris) Editorial

Aufsätze

316 Hilke ElSEN (MüNCHEN)

Affixoide: Nur was benannt wird, kann auch verstanden werden

352 HEIDRUN KäMPER (MANNHEIM)

Demokratiegeschichte des 20. Jahrhunderts als Zäsurgeschichte das Beispiel der frühen Weimarer Republik. Ein transdisziplinäres Forschungsprojekt.

334 Sascha Michel (Koblenz)

Schaden-0-ersatz vs. Schaden-s-ersatz. Ein Erklärungsansatz synchroner Schwankungsfälle bei der Fugenbildung von $\mathrm{N}+\mathrm{N}$-Komposita

83 Vedad Smailagić (Sarajevo) Valenzänderung

289 ANJA StUKenbrock (FreIbURG)

Referenz durch Zeigen: Zur Theorie der Deixis

\section{THEMENHEFT: SPRACHE IN OST UND WEST}

95 Albrecht Plewnia (Mannheim) Sprache in Ost und West

97 HaRTMUt Schmidt (MANnheim) Über den gemeinsamen Sprachgebrauch in Ost und West, seine Probleme und kreativen Möglichkeiten

130 NORBERT RichARD WOLF (WÜRZBURG)

Der ,DDR-Wortschatz، als Indikator einer nationalen Varietät?

Mit einem Blick auf die Lexikographie des DDR-Wortschatzes

148 DoRis STEFFens (MANNheim)

20 Jahre Mauerfall - Zur Wortschatzentwicklung seit der Wendezeit

168 Friedhelm Debus (Kiel)

Namen in Ost und West

182 Hans-Werner Eroms (Passau)

Eigen- und Fremdbenennungen im Deutschland der Wende- und Nachwendezeit

206 Manfred W. Hellmann (Mannheim)

Kontroversen um das "sprachliche Ost-West-Problem“.

Zum Spannungsfeld zwischen Politik, Wissenschaftsförderung und Sprachwissenschaft.

Ein forschungshistorischer Rückblick 
235 Albrecht Plewnia (Mannheim)/Astrid Rothe (Mannheim)

Eine Sprach-Mauer in den Köpfen? Über aktuelle Spracheinstellungen in Ost und West

THEMA: POLYSEMIE

3 Kristel Proost (Mannheim)/Edeltraud Winkler (Mannheim) Vorbemerkung

5 Klaus-Peter Konerding (Heidelberg) Unterspezifikation, Sorten und Qualia-Rollen: Skizze eines integrativen Modells

33 Edeltraud Winkler (MANNheim)

Überlegungen zu Artefaktbezeichnungen im Deutschen

48 KLaus von Heusinger (STUtTGart)

Zur Bedeutung von Derivationen an der Schnittstelle von lexikalischer Semantik und konzeptueller Struktur

65 Kristel Proost (Mannheim)

Bedeutung und Standardinterpretation von Äußerungen mit negierten negativ-bewertenden Adjektiven

Dokumentationen

280 Michael Hoffmann (Potsdam)

Zwei Reader zur Forschungsgeschichte. Eine Rezension

370 AlEXANDER LASCH (KIEL)

Die Diskurslinguistik nach Foucault und die epistemischen Dimensionen sprachlicher Sachverhaltskonstitution. Eine Doppelrezension

379 Beate Lingnau/Birte Schaller (Bielefeld)

Bericht über das 43. Treffen des „Arbeitskreises angewandte Gesprächsforschung“" am 14. und 15. November 2008 in Koblenz

287 Notizen 


\title{
Affixoide: Nur was benannt wird, kann auch verstanden werden ${ }^{1}$
}

\begin{abstract}
Im Mittelpunkt des Artikels stehen die sogenannten Affixoide bzw. Halbaffixe sowie ihre positionsbedingten Entsprechungen Präfixoid/Halbpräfix und Suffixoid/Halbsuffix, häufig genannte Kandidaten sind arm, fri, leer, voll, riesen, affen, mords, bomben. Der Schwerpunkt liegt hier neben den Begriffen vor allem auf d:r Kategorie. Nach einem kurzen Abriss zur Entstehung des Affixoid-Konzepts im deutschsprachigen Raun werden einige unterschiedliche Ansichten dazu vorgestellt. Es folgt die Erörterung verschiedener Grünce für die Vorzüge einer solchen Kategorie.

The present article focuses on so-called affixoids/semi-affixes and their counterparts prefixoids/semi-prefixes and suffixoids/semi-suffixes respectively in German. Frequently quoted examples are arm, frei, leer, vol, riesen, affen, mords, bomben. However, the discussion does not focus on terminology, but rather on the concept itself. After a short review of early approaches, several diverging views are presented, followed ty a discussion of arguments in favour of the concept.
\end{abstract}

\section{Frühe Beobachtungen zur Übergangszone zwischen Derivation und Komposition}

Der Grenzbereich zwischen Komposition und Derivation wird nicht erst heute beschrieben, und zwar nicht nur entwicklungsgeschichtlich, denn viele Affixe entstanden aus Kompositionsgliedern (vgl. westgerm. -bāri- 'tragend, hervorbringend', ahd. -bāri, nhc. -bar, germ. -likka-, got. leik 'Körper', nhd. -lich, got. haidus 'Art, Weise' nhd. -heit, Krahe/ Meid 1967, S. 15, 48, 220). Nach Jacob Grimm (1826, S. 1231 in Lachachi 2008, S. 221) stellt auch Wilmanns (1899, S. 555 f.) fest, dass es Komposita gibt, in denen das erste Glied den semantischen Kern bildet und das zweite, wie -werk oder -zeug, abstrakt und allgemein sich einer Ableitungssilbe nähert. Paul (1937, S. 348) weist darauf hin, dass die Scheidelinie zwischen Suffix und Kompositionsglied nur durch das Sprachgefühl bestimmt werden könne. Bei einem Affix verliert ein Kompositionsglied „die Fühlung“ mit dem ursprünglich identischen einfachen Wort, beide müssen dabei etymologisch verwandt sein, das Element muss nicht nur vereinzelt, sondern in einer Gruppe von Wörtern in jeweils gleicher Bedeutung vorkommen und das Kompositionsglied entwickelt dann eine gewisse abstrakte Allgemeinheit (Paul 1937, S. 347). Manche Kompositionsglieder wie in anmutsvoll, schmerzvoll nähern sich ,dem Charakter“ eines Suffixes (Paul 1937, S. 348).

Henzen (1957) bestätigt, dass die Komposition oft lediglich zur Verstärkung dient (Höllenqual, Sauglück, riesengroß, stockfinster, Henzen 1957, S. 63, 65), dass ein Glied lediglich rein mechanisch aus einer anderen Bildung übernommen wird (blitzdumm, hundekalt, blutjung, ibd., S. 65) und dass es zu immer zahlreicher werdenden gruppenartigen Bildungen kommt (ibd., S. 192, 209 f.). Gewisse zweite Glieder wie voll, los, reich etc. werden mit der Zeit so geläufig, dass sie zu den Ableitungen „hinneigen“ (ibd., S. 65). Die

Die Arbeit entstand im Rahmen des Projekts Deutsche Wortbildung, LMU München, das dankenswerterweise gefördert wird durch Seidl Vermessung und die DFG. 
Zusammensetzungen nähern sich deswegen den Ableitungen (gemeint sind Suffigierungen), weil das zweite Glied nicht mehr das Hauptgewicht des Wortsinns trägt (Laubwerk, Schreibzeug, ibd., S. 192). Eine Einheit wird schließlich als Suffix empfunden, wenn das selbstständige Wort untergeht (ibd., S. 110). Damit führen Paul und Henzen charakteristische Eigenheiten bestimmter Elemente an, die sie von Kompositagliedern und Affixen unterscheiden und so zu einem Zwischenstatus führen: Sie existieren auch als freies Wort, aber wiederholt mit Bedeutungsverlust im zusammengesetzten Wort, Reihenbildung. Sie verweisen darüber hinaus auf den Weg freier Wörter zum Affix (Paul 1937, S. 347 ff., Henzen 1957, S. 109, 111), auch im Zusammenhang mit Grammatikalisierung (Henzen 1957, S. 202). Insgesamt war also das Problem der klaren Trennlinie zwischen Komposition und Derivation in den damals noch hauptsächlich diachron ausgerichteten Arbeiten stets bewusst.

\section{Argumente für einen Begriff}

Während Naumann (1972, S. 6, 9) noch anhand von Schreibzeug vs. Viehzeug bzw. haupt auf den Problembereich zwischen Derivation und Komposition ohne eigenen Begriff hinweist, diskutiert Petermann (1971) zahlreiche Beispiele, die sich nicht eindeutig als Präfigierung oder Zusammensetzung analysieren lassen, mit systematischen Gemeinsamkeiten und schlägt für sie den Begriff Halbpräfix vor. Fleischer (1982, S. 70) fordert dann ausdrücklich, für die Gruppe der Einheiten in dieser Übergangszone, die das aus der Prager Schule bekannte Problem von Zentrum und Peripherie veranschaulichen, einen eigenen Terminus zu verwenden. In den 70er und 80er Jahren gebrauchen mehrere Autoren die Begriffe Affixoid bzw. Halbaffix in ihren Arbeiten zur Wortbildung des Deutschen, neben Fleischer auch Stepanova und Kühnhold et al. (vgl. den Überblick in Tellenbach 1985). In der weiteren Diskussion versucht Vögeding zu zeigen, dass es zwar keine klare Trennungslinie zwischen Derivation und Komposition gibt, dass sich aber Halbsuffixe durch kompositionsgliedtypische Phonologie und Isolation der Funktion bzw. Semantik von Suffixen und Kompositionsgliedern gleichermaßen abheben und dass daher eine Dreiteilung gerechtfertigt ist, da durch sie eine Trennung klarer wird (Vögeding 1981, S. 111). Für Tellenbach (1985) entwickelt die Zwischenkategorie deswegen eine gewisse Relevanz, weil entsprechende Einheiten in die Bedeutungswörterbücher aufgenommen werden sollten mit Angaben zur Verwendung, einerseits, um die Menge der Stichwörter zu reduzieren, andererseits, um den Benutzern Informationen zu Gebrauchsregularitäten zur Verfügung zu stellen. Insofern bekam die Notwendigkeit, durch einen Begriff auch eine Kategorie zu etablieren, mehr und mehr Relevanz.

\section{Kriterien}

Petermann (1971) stellt in Fortführung und Systematisierung der älteren Beobachtungen die wichtigsten drei Merkmale der Präfixoide zusammen, die u.a. Tellenbach (1985) auf Suffixoide überträgt.

\subsection{Bedeutung}

Das Präfixoid weist statt einer lexikalischen Bedeutung verstärkende, intensivierende, wertende Bedeutungsaspekte auf, Suffixoide oft kollektive, privative. Schon früh wurde konstatiert, dass im Gegensatz zu Determinativkomposita wie Autowerk das Erstglied die Hauptbedeutung des Gesamtausdrucks bestimmt - ein Autowerk ist eine Art Werk, Schuh- 
werk hingegen sind Schuhe. Das Bestimmungswort ist zum semantischen Kern gewor$\operatorname{den}^{2}$ und die Paraphrase *'Werk aus Laub' für Laubwerk ist nicht akzeptabel. Die Kollektiva Backgut, Gedankengut, Menschengut stehen Landgut bzw. Gut 'Vermögen' gegenüber. ${ }^{3}$

Tellenbach (1985, S. 286 f.) präzisiert in Anlehnung an Fleischer (1982): Während Kompositionsglieder eine klare Einzelbedeutung aufweisen, sind Affixoide stärker abstrahierend, weniger konkret, dafür kategorieller, allerdings nicht ganz so verallgemeinernd wie Affixe. Metaphorischer bzw. vergleichender Gebrauch der Einheiten reichen ihr jedoch nicht aus, um sie als Affixoid zu klassifizieren. Damit hängt das nächste Merkmal eng zusammen.

\subsection{Gleichlautendes selbstständiges Wort}

Die Einheit existiert auch als freies Wort; beide müssen etymologisch verwandt sein (Stepanova in Schmidt 1987b, S. 58 f.). Die Bedeutungsbeziehung zwischen beiden geht verloren. Tellenbach (1985) möchte zwar noch einen Zusammenhang sehen, eine Paraphrase mit dem freien Wort sollte aber nicht mehr möglich oder nicht sprachüblich sein: Ein fischarmer Fluss ist kein 'Fluss, der arm an Fischen ist', sondern 'ein Fluss, in dem wenig Fische sind'. Noch deutlicher wird dies am Beispiel pflegearme Motorfahrräder (Tellenbach 1985, S. 293 f.). Vögeding lässt sowohl Homonymie wie bei artig $^{4}$ als auch Bedeutungsverwandtschaft wie bei los gelten, solange „ein gebundenes Element Leistungen vollbringt, die es in ungebundener Form jeweils nicht erfüllen kann“ (Vögeding 1981, S. 112).

Im Folgenden wird die etymologische Verwandtschaft zwischen der freien und gebundenen Variante vorausgesetzt. Wenn die Paraphrase die beteiligten Lexeme enthält und die Relationsbedeutung deutlich macht, weist dies auf die Motiviertheit der Bildung hin und spricht gegen eine Affixoidinterpretation. Dies ist jedoch, wie später gezeigt wird, auf die Gruppe einer Affixoidbildung insgesamt zu beziehen.

\subsection{Reihenbildung}

Die Einheiten sind produktiv und bilden größere Gruppen. Als Gegenargument wird häufig eingewendet, dass sich auch einige Kompositaglieder reihenbildend verhalten können. Aber dies gilt nur lexembedingt für Einzelwörter und unter Beibehaltung der Eigenbedeutung. Korrekter wäre wohl eine Formulierung, die auf eine grundsätzliche bzw. automatische Reihenbildung abzielt.

2 Dazu bemerkt Vögeding (1981, S. 73 f.), auch bei Derivaten sei eine Determination des zweiten durch das erste Glied möglich, aber es fehlen Beispiele. Hartmann/Hansen (1991, S. 71 f.) behaupten, bei Suffixbildungen, z.B. mit -er, läge der semantische Kern auch nicht auf dem Erstglied. Hier liegt ein anderes Verständnis von Kern vor.

3 Die Argumente führen Fleischer (1982, S. 69) zu einer Zuordnung von werk und gut zu den Affixen. Fleischer/Barz (1995) bleiben bei werk als Affix, gut jedoch wird zu einem Kompositionsglied.

4 Laut Kluge (1999) heißt dies eigentlich 'von guter Art' und ist durchaus bedeutungsverwandt mit Formen wie in sturzflutartig. 


\subsection{Weitere Kriterien}

Kühnhold et al. führen außerdem kombinatorische Charakteristika auf, da ein Suffixoid ohne Erstglied, wie im Falle der Affixe, nicht verwendbar ist (hautfreundliche Seife * freundliche Seife, Kühnhold et al. 1978, S. 427 in Schmidt 1978b, S. 61, vgl. auch Fleischer/Barz 1995, S. 27 liebevolle Geste - *volle Geste). Dieses Merkmal bleibt umstritten.

Die Autorengemeinschaft um Kühnhold fasst weiterhin die Konkurrenz mit Affixen als Merkmal auf (Schmidt 1987b, S. 61), was Tellenbach (1985) als Funktionsgemeinschaft der Affixoide mit Affixen bezeichnet. Allerdings kann Schmidt dieses Argument zu Recht entkräften, denn es gilt genauso für manche Kompositionsglieder (Zimmermann / Zimmerer, Schmidt 1987b, S. 63, in einem anderen Zusammenhang Fußballspieler / Fußballer, Vögeding 1981, S. 74).

Petermann (1971) verweist auf ein weiteres Kriterium, nämlich auf die Akzentverhältnisse in Determinativkomposita im Vergleich zu den von ihm beschriebenen Beispielen im Determinativkompositum wird das erste von zwei Gliedern betont, in Bildungen mit Präfixoiden beide oder das zweite: 'blutarm' vs. 'blut'arm, 'bombensicher' vs. 'bomben'sicher (Petermann 1971, S. 111). Entsprechend würde 'Riesenparty' als 'Party mit Riesen' aufgefasst, 'Riesen'party oder Riesen' party als 'sehr große Party', ein 'Bombenfund' ist der ,Fund einer Bombe', ein 'Bomben'fund ein 'außergewöhnlicher Fund'. Dieses Argument wird in der Folge als Kriterium für die Unterscheidung von Präfixoidbildungen und (Determinativ)Komposita fast nie wieder aufgenommen ${ }^{5}$, eine Ausnahme bildet u.a. Ascoop (2005).

\subsection{Kriterienbündel}

Ausschlaggebend aber für die Bestimmung ist die Bündelung der Kriterien Reihenbildung, semantische Veränderung und freies Pendant (Elsen 2004, S. 30).

Da das Affixoid von der Kategorie Kompositionsglied in die oppositionale Kategorie Affix eindringt, sind von einem Affixoid, wenn auch nicht in vollem Maße, so doch bis zu einem gewissen Grad die Kriterien eines Affixes zu erfüllen. Dabei müssen alle Kriterien zusammenwirken. (Tellenbach 1985, S. 294)

Auch Kompositaglieder können Reihen bilden, aber sie behalten ihre Eigenbedeutung bei; Haustüren, Wohnungstüren, Fabriktüren sind immer Türen. Schuhwerk, Astwerk oder Laubwerk haben nichts mehr mit einem Werk zu tun.

Der letzte Punkt verschwindet in den späteren Diskussionen ebenfalls. Denn nach den dem Begriff positiv eingestellten Arbeiten gehen die Ansichten in den späten 80er und 90er Jahren in Opposition dazu. In den Argumentationen werden die Kriterien in Isolation geprüft und als distinktive Eigenschaften verworfen. Da die zu prüfende Kategorie keine ihr eigenen distinktiven Eigenschaften aufweist, so die Folgerung, ist keine Kategorie anzusetzen.

\footnotetext{
Hansen/Hartmann (1991, S. 115) stellen zwar eine besondere Akzentverteilung fest, führen dies aber auf
} semantische Gründe zurück und ordnen bombensicher und stinksauer bei den Präfixbildungen ein. 


\section{Argumente gegen einen Begriff}

Interessanterweise wird immer wieder konstatiert, eine klare Grenzziehung zwischen Komposition und Derivation sei nicht möglich, von Grimm über Wilmanns, Paul, Vögeding (1981), Hansen/Hartmann (1991, S. 85, 161) bis hin zu Fleischer/Barz (1995) u.a. Trotzdem bleibt das Plädoyer für diese Zweieraufteilung relativ weit verbreitet. Die Argumente hierfür zielen stets darauf ab, ein Begriff wie das Affixoid sei nicht nötig, durch ihn sei nichts gewonnen. Es sei zu entscheiden zwischen Komposition und Derivation (u.a. Schmidt 1987b, Fleischer/Barz 1992).

Als Grundlagenarbeit gegen die Affixoide dient Schmidt (1987b). Er fasst die Verwendung eines eigenen Begriffs als Versuch auf, sich einer Zuordnung zu Affix oder Grundmorphem zu entziehen und stellt dessen Brauchbarkeit und Notwendigkeit in Frage. Schmidt lehnt unnötige Zusatzklassen und -begriffe ab, um zu viele Unterklassen zu vermeiden. ${ }^{6}$ Es sei in jedem Fall zwischen der einen oder anderen Wortbildungsart zu entscheiden. In der Folge stützen sich die meisten, die sich gegen den Affixoidbegriff entscheiden, auf seine Argumentation. Darum sollen die wichtigsten Aspekte hier behandelt werden.

\subsection{Semantische Unklarheiten}

Das erste Problem bietet der Affixoidbegriff hinsichtlich der inhaltlichen Bestimmung. Eine semantische Abweichung zum freien Lexem ist nicht immer klar entscheidbar (Schmidt 1987b, S. 73). Oft werden auch geringe Abweichungen von den freien Wörtern als Anlass genommen, sie zu den Affixoiden zu zählen (ibd., S. 74). Ihr semantischer Gehalt wird von Autor zu Autor eher kleiner, eher größer gesehen (ibd., S. 77), die semantische Identität unterschiedlich eng (ibd., S. 78). Diese Kritikpunkte sind jeder für sich stichhaltig. Sie sind allerdings erstens den Arbeiten einzelner Autoren zuzuordnen und nicht grundsätzlich dem Affixoidbegriff, obwohl sie natürlich die damit verbundenen Unsicherheiten aufzeigen. Zweitens aber treten solche Differenzen häufig bzw. typischerweise in Diskussionen zu neuen Ideen auf. Es dauert stets eine gewisse Zeit, bis Einigkeit zu Definitionen herrscht und sich verschiedene Lager auf ihre jeweiligen Standpunkte einigen. Das Bedeutungskriterium ist sicherlich nicht ganz unproblematisch. Die damit verbundenen Schwierigkeiten reichen aber nicht aus, um den Affixoidbegriff zu verwerfen, denn sie führen auch ohne ihn zu Differenzen bei den Analysen.

In diesem Zusammenhang ist der Paraphrasetest zu sehen, der, so sei an dieser Stelle betont, natürlich nur der Überprüfung semantischer Verhältnisse dient, nicht als eigenständiges Kriterium. Der Paraphrasetest also sollte bei Affixoidbildungen auf die Verwendung des freien Wortes verzichten. Schmidt wendet dagegen ein, dass die Syntax von Wortbildungen und Wörtern im Satz unterschiedlichen Gesetzen gehorchen und daher nicht vergleichbar sei (ibd., S. 85). Sein Einwand trifft für diejenigen Bildungen zu, die nicht über die Zusammensetzung zweier Grundmorpheme, sondern über Ableitung entstanden sind wie trinkfreudig als Adjektivierung von *Trinkfreude oder schreiblustig aus Schreiblust. Freudig und lustig können darum nicht in Paraphrasen als freudig und trinken bzw. lustig und schreiben erscheinen und eignen sich nicht als Testkriterium für den Suffixoidstatus

6 Zu diesem Problembereich zählt auch der Begriff des Konfixes; zu Diskussion und Abgrenzung von Konfix und Affixoid vgl. Elsen (2005b). 
der Adjektive (ibd., S. 85 f.). Diese Argument lässt sich jedoch nicht auf Beispiele wie fisch-oder pflegearm übertragen.

\subsection{Eine zusätzliche Kategorie bringt keine Vorteile}

Schmidts zweites Argument richtet sich darauf, dass Zuordnungsschwierigkeiten durch eine Zwischenkategorie größer werden (ibd., S. 78) und dass neue Kategorien wegen kleiner Unterschiede keinen Erkenntnisgewinn bringen, wenn sie „nicht aus der Natur der Sache abgeleitet sind, sondern nur durch das Bedürfnis nach Ordnung oder intellektueller Durchdringung legitimiert werden können“ (ibd., S. 85). Außerdem gibt es aufgrund des zusätzlichen Begriffs nicht einen, sondern zwei Übergänge (ibd., S. 98).

Nun lehnt Schmidt in der Affixoiddiskussion zwar unnötige Zusatzklassen und -begriffe $\mathrm{ab}$, um zu viele Unterklassen zu vermeiden. In seiner Arbeit im gleichen Band zu „Kombinem“, „Komponem“, „Basem“, „Basokomponem“, „Präponem“ usw. (Schmidt 1987a) aber entscheidet er sich genau entgegengesetzt und führt diverse zusätzliche Kategorien ein, wenn nicht zwischen Affix und freiem Grundmorphem entschieden werden kann. Die weiteren Begriffe „Präpostponem“, „Basopräponem“, „Basopräpostponem“ lassen schließlich sein ursprüngliches Argument unglaubwürdig erscheinen.

Auch in anderer Hinsicht kann an der Ernsthaftigkeit seiner Darstellung gezweifelt werden. Wenn er zu Ornter/Ortner Stellung nimmt mit ,,so führen sie den Begriff (oder den Begriffoid?) ,affixoidnahes Element' ein“ (ibd., S. 70), dann wirkt „Begriffoid“ nicht wissenschaftlich, und die Kritik an dem zusätzlichen Terminus geht angesichts der Basopräponem-Vorschläge ins Leere. Weitere nicht ganz wissenschaftliche Formulierungen folgen. Schließlich kritisiert er Kienpointer:

So entsteht der Eindruck, als wollte sie alle WB-Konstituenten, die im Bereich der freien Wörter lautgleiche Entsprechungen haben, aber inhaltlich und auch in anderer Weise von diesen abweichen, zu den Suffixoiden zählen, was schon angesichts der Fälle Freund-schaft, bos-haft, Maler-ei usw. nicht so ohne weiteres akzeptabel ist. (Schmidt 1987b, S. 67).

Dabei ist ernsthaft zu bezweifeln, ob jemand $E i$ als Suffixoid betrachten würde. Die Ausführungen sind damit weder ganz seriös noch stichhaltig. Seine Erkenntnis, eine Übergangszone sei besser als zwei (ibd., S. 98, vgl. auch Donalies 2002, S. 26), kommt zwar einem überzeugenden Argument noch am nächsten. Aber, wie gleich noch gezeigt wird, sind mit einer einzigen Übergangszone genügend Probleme verbunden, um die Alternative mit zwei Übergangszonen zu prüfen. Außerdem geht es heute, über zwanzig Jahre später, nicht mehr allein um diesen Übergang, sondern um die stetig wachsende Gruppe sich ähnlich verhaltender Elemente, weder klar Komposita noch eindeutig Ableitungen, die eine gesonderte Beachtung verdient. 
Insgesamt sind die Gründe, die Schmidt gegen die Verwendung des Affixoidbegriffs anführt, nicht einsichtig, die Diskussion weist wiederholt polemische Züge auf. Auf Petermanns (1971) Ausführung zu den unterschiedlichen Akzentverhältnissen geht er gar nicht ein und auch die Forderung nach dem Kriterienbündel ignoriert er.

\section{Unterschiedliche Standpunkte}

\subsection{Weitere ablehnende Arbeiten}

Fleischer/Barz (1995, S. 27 f.) geben Schmidt verkürzt und fragmentarisch wieder und lehnen den Affixoidbegriff ab. Gegen den Terminus stellen sich auch Altmann/Kemmerling (2000), Lohde (2006) und Donalies (2002, S. 25 f.), dort unter Verweis auf die komplizierteren Verhältnisse und die fehlende Erklärungskraft dieser Kategorie. Auch Eisenberg (1998, S. 210) ist gegen den Terminus, denn die meisten Bildungen seien formal als Komposita zu behandeln (anders aber dann Eisenberg 2006). Laut Eichinger (2000, S. 87) sollten statt des Begriffs besser besondere Arten von Derivation bzw. Komposition angesetzt werden. Olsen (1986) bezeichnet den Terminus als überflüssig, da er nichts zur Erklärung vor allem der Reihenbildung beitrage. Für Hansen/Hartmann (1991) ist die Kategorie auch nicht nötig; sie setzen aber neben der Komposition zwei Klassen an Suffixen, worttypische und suffixtypische, an. Auch Fandrych (1993) erachtet die Kategorie als entbehrlich und übernimmt damit Schmidts Schlussfolgerung, allerdings nach einer sehr ausführlichen Studie zu adjektivischen Suffixoidkandidaten, für die eine solche Kategorie mehr Gemeinsamkeiten verdecke als Erklärungskraft besitze (Fandrych 1993, S. 101).

Sie alle sind der Auffassung, es sei in jedem Fall zwischen Derivation und Komposition zu entscheiden. Ausführliche Auseinandersetzungen wie bei Olsen und Fandrych sind dabei selten, oft werden entweder keine Begründungen gegeben oder es werden einige Ansichten Schmidts wiederholt. Wenn es zu Diskussionen kommt, erfolgt die Beleuchtung der Kriterien stets in Isolation. Sie sind jedes für sich dann nicht ausreichend, um eine eigene Kategorie Affixoid zu definieren und zu rechtfertigen.

Ein weiteres Argument stammt von Vögeding, der allerdings dem Begriff positiv gegenübersteht. Ihm fällt auf, dass keine eigenen Termini für entsprechende Wortbildungsprozesse erscheinen, also gibt es offenbar kein eigenes Wortbildungsverfahren, vielmehr sind die Morpheme selbst für den Übergangscharakter verantwortlich (Vögeding 1981, S. 75). Und Fandrych schließlich stört es, dass der Begriff nur durch negative Definition gewonnen wird und dass eine positive Charakterisierung unklar bleibt (Fandrych 1993, S. 1, 74).

\subsection{Neutrale Standpunkte}

Neben der ausdrücklich ablehnenden Haltung gibt es einige Autoren, die den Begriff zwar erwähnen, die Beispiele aber trotzdem den Komposita oder den Derivationen zuordnen. Der Duden behandelt die entsprechenden Formen bei den Komposita, die mit gebundenen Gliedern gebildet sind. Im Kapitel zur Derivation werden dann jedoch bestimmte Affixe funktional ergänzt durch Wörter (Barz 2006, S. 741) - dem Leser bleibt es überlassen, dies als Komposition zu werten oder als Derivation. Motsch (1996) interpretiert artig, förmig, farbig, haltig als aus Derivation entstanden, frei als Kompositionsglied. Der Terminus Affixoid ist seiner Ansicht nach sinnvoll ,als Sammelbezeichnung für einen Bereich von Wortbildungserscheinungen“" (Motsch 1996, S. 161, auch Ascoop 2005, Decroos/Leusch- 
ner 2008, Leuschner i. Dr.). Genauso gut können die meisten Beispiele mit Affixoiden aber als Komposita oder besondere Derivationen bezeichnet werden. Später ordnet er artig, farbig und frei bei den Affixen mit Sonderstatus ein (Motsch 1999, S. 12, 274).

\subsection{Konstruktive Verwendung des Begriffs}

Positiver dem Begriff gegenüber eingestellt sind beispielsweise Erben (1993) oder Bergmann/Pauly/Stricker (2001). Eine selbstverständliche Anwendung findet sich u.a. in Lühr (1996), Simmler (1998) und Kluge (1999). Engel (1988) behandelt die Elemente nur bei der adjektivischen Wortbildung. Er nennt Präfixoide ursprünglich selbstständige Wörter, die wie Präfixe verwendet werden. Sie behalten ihre Bedeutung bei oder nehmen eine neue an (Engel 1988, S. 578). Die stark reihenbildenden Suffixoide weisen eine verblasste, aber noch erkennbare Bedeutung auf (Engel 1988, S. 579). Bei den Nomen gelten haupt, blitz, bomben etc. als Präfixe (Engel 1988, S. 512).

Für Hansen/Hartmann (1991, S. 41) setzt die konsequenteste Konzeption einen eigenständigen Wortbildungsprozess an, sie stellen aber fest, dass dieser Gedanke sich nicht durchsetzen konnte. Das ist mittlerweile anders. In Elsen (2004) wurde gezeigt, dass das quantitative Verhalten von Affixoidbildungen in verschiedenen Varietäten des Deutschen schwankt und dass daher eine Abgrenzung zu anderen Wortbildungsarten durchaus sinnvoll ist, wenn Zusammenhänge zwischen Gebrauchsfunktion bzw. Sprecherintention und Wortbildungsverfahren untersucht werden. Für Stevens (2005) handelt es sich um ein nützliches Konzept, vor allem aus diachroner Sicht. „In sum, affixoids are more grammaticalized than roots, and affixes are more grammaticalized than affixoids" (Stevens 2005, S. 78). Ascoop (2005) benötigt die Kategorie für sprachvergleichende Untersuchungen und Lachachi (2008, S. 225) betrachtet die Halbaffigierung ausdrücklich als eine Wortbildungsart, da sie sehr produktiv geworden ist.

Zusammengefasst gibt es heute die folgenden vier Positionen zum Affixoid. (1) Es ist zwischen Derivation und Komposition zu entscheiden. (2) Der Begriff wird erwähnt, fließt aber nicht produktiv in eine Behandlung ein, sondern die Beispiele werden entweder den Komposita oder den Derivaten zugerechnet. (3) Das Affixoid ist zwar keine Kategorie, aber ein nützlicher Begriff. (4) Und schließlich bildet das Affixoid ein eigenes Konzept und neben den Wortbildungsarten Derivation und Komposition wird auch Affixoidbildung (Halbaffigierung) angesetzt.

\section{Probleme bei einer Zweierteilung in Komposition und Derivation}

Der Begriff und seine Anwendungen waren in der Vergangenheit mit einigen Unklarheiten verbunden. Dabei gingen die Schwierigkeiten, die bei morphologischen Analysen ohne ihn auftreten, ganz unter.

\subsection{Werkintern unterschiedliche Zuordnung}

Einerseits kommt es nämlich zu unterschiedlichen Einordnungen einer Einheit innerhalb eines Werkes. Beispielsweise zählen Fleischer/Barz Bildungen mit haupt einmal zur Komposition, dann aber wird es als Präfix bezeichnet (Fleischer/ Barz 1995, S. 101, 205). Auch bei Lohde ist es einmal ein „kompositionelles Erstglied“, einmal Präfix (Lohde 2006, S. 
65, 147). Barz behandelt riesen und mords bei den Nomen als Affix, bei den Adjektiven als Kompositionsglied (Barz 2006, S. 741, 756). Nun bezeichnet zwar Ascoop (2005, S. 22) die Bildung riesengro $\beta$ als unproduktive Ausnahme und die seltenen riesenlang oder riesenstark als eher ungewöhnlich. Das spricht für den Status einer Metapher bei dem Adjektiv ,groß wie ein Riese' und damit für Komposition, aber nicht gegen die Einordnung als Präfixoid bei den Nomen.

\subsection{Werkübergreifend unterschiedliche Zuordnung}

Weiterhin entscheiden sich die verschiedenen Autoren für unterschiedliche Einordnungen. Dies sollen lediglich einige Beispiele veranschaulichen. Barz behandelt spitzen, monster, haupt, höllen, mammut, affen, heiden, sau bei der Derivation, grund, brand, grotten, hoch bei der Komposition (Barz 2006, S. 741, 756). Für Hansen/Hartmann (1991, S. 111, 115, 124) sind haupt, affen, bomben, stink, stock, sau, ober Präfixe. Bei Olsen (1986) und Fandrych (1993) sind frei, arm, leer, voll, reich Kompositionsglieder. Reihenbildende Grundmorpheme sind bei Fleischer/Barz (1995) arm, voll, reich, frei, grund, riesen, hoch, bomben, affen, sau, monster, gut, stelle, zeug, fähig, fest. Affixe sind los, werk, wesen, mäßig. Motsch (1999) behandelt artig, farbig, farben, mäßig und förmig als Suffixe. Lohde interpretiert ober, riesen, sau, heiden, bomben, grund, hoch, voll, tief, fähig, freudig, arm, sicher, voll oder artig als Kompositionsglieder, werk, wesen, haupt, halben, weg, weise und mäßig als Affixe.

Es ist nicht nachzuvollziehen, warum monster ein Affix, aber grotten ein Kompositionsglied sein soll oder warum sau, mords, affen, artig und bomben einmal als Affix, ein andermal als Kompositionsglied behandelt werden. Der Vorwurf, bei einer Zwischenkategorie käme es zu unklaren Einordnungen, der ja, völlig zu recht, schon bei Schmidt (1987) $\mathrm{zu}$ lesen war, trifft also genauso gut auch für die Zweierteilung zu.

\subsection{Inkonsequenzen in der Argumentation}

Einerseits wird der Hinweis auf den Zwischenstatus häufig wiederholt, etwa „Damit kommt Riese(n)- dem Status eines Präfixes am nächsten, ohne ihn jedoch ganz zu erreichen“ (Fleischer/Barz 1995, S. 100) oder „Darunter befinden sich jedoch auch einige Zweitglieder wie -fähig, -freudig oder -arm, welche aufgrund ihrer Gebrauchsweise zweifellos zum Status eines Suffixes tendieren" (Lohde 2006, S. 158). Trotzdem wird die Forderung nach einer Zweiteilung aufrechterhalten. Wesentlich unverständlicher jedoch ist, dass manche Autoren, die die Kategorie und den Begriff ablehnen, stattdessen andere Termini verwenden, z.B. „gebundene Wurzeln“ (Fandrych 1993, S. 107). Es scheint, dass zumindest für die Diskussion ein Wort gebraucht wird. Auch Barz spricht von Komposita mit gebundenen Zweitgliedern (Barz 2006, S. 768). Das zeigt einerseits den besonderen Status (die Glieder sind nur im Kompositum gebunden, denn sonst sind sie frei), andererseits auch den Bedarf nach einem Terminus für die entsprechenden Einheiten. Altmann/ Kemmerling (2000) gehen sogar so weit, den Begriff des Affixoids zwar dezidiert abzulehnen, aber einen weiteren Wortbildungstyp neben Determinationskomposition und Präfigierung einzuführen, die Steigerungsbildung (affengeil, mausetot, wortwörtlich, Affenhitze, Mordsweib). Demnach sind sattgrün und tiefblau keine Komposita, ultraleicht ist keine Ableitung (ibd., S. 35). Inkonsequent ist besonders, dass diese Kategorie im Gegen- 
satz zu den anderen Wortbildungsarten nicht auf strukturellen, sondern rein auf semantischen Kriterien basiert und manche Präfixbildungen mit Komposita auf inhaltlicher Grundlage vereint. Fandrych schließlich beschreibt die Gruppe um -frei, -arm, -voll, -leer und -reich als fast rein funktionale Elemente, die Bildungsmuster als kohärent, die jeweils realisierten semantischen Relationen als uniform (Fandrych 1993, S. 113 f.). Er nennt sie lexikalische Junktoren (Fandrych 1993, S. 116) und fasst sie über gemeinsame Eigenschaften und mithilfe des Terminus zu einer Kategorie zusammen. Später geht er auf Unterschiede zwischen Junktionsbildungen und freien Entsprechungen bzw. Suffixen ein (Fandrych 1993, S. 244 f.). Eine den Affixoiden vergleichbare Kategorie existiert damit auch hier, nur wird sie anders bezeichnet.

Probleme ergeben sich also in der Regel dadurch, dass einerseits Zuordnungen und Kriteriengewichtungen inkonsequent eingehalten und Entscheidungen für die eine oder andere Wortbildungsart nicht einheitlich getroffen werden, was auf Unsicherheit bzw. unklare Verhältnisse hinweist und dadurch als das überzeugendste Argument gegen die Zweiereinteilung zu werten ist. Unterschiede werden durchaus anerkannt, die Fixierung soll aber lediglich anhand von Umschreibungen oder zusätzlichen Kommentaren erfolgen. Andererseits wird sogar eine weitere Kategorie eingeführt, das macht die Unvollkommenheit der Zweieraufteilung noch offensichtlicher. Die Einordnung einer Bildung in entweder Kompositum oder Derivation ist weiterhin auch deswegen unzufriedenstellend, weil Beispiele wie bettelarm, bitterarm, wartungsarm, bügelarm auf der Wortbildungsebene gleich zu behandeln sind. Die Unterschiede werden nivelliert und höchstens durch zusätzliche Erklärungen und kontrastive Paraphrasen deutlich. Schließlich weisen viele Autoren trotzdem wieder so häufig auf Grenzfälle oder unklaren Status hin, dass sich eine weitere Kategorie geradezu aufdrängt.

\section{Affixoid als Kategorie}

Um diese Position zu stützen, sollen die in der Vergangenheit gefundenen Kriterien noch einmal zusammengestellt werden.

Aus klassischer Perspektive sind Kategorien diskret und werden durch eine Gruppe von Merkmalen charakterisiert, über die alle Mitglieder in gleicher Weise verfügen. Im Gegensatz zu diesen an Aristoteles orientierten strukturalistischen bzw. generativen Vorstellungen werden hier allerdings Eigenschaften als mehr oder weniger treffend bzw. gut verstanden. Vor allem Kriterienbündel dürfen statt lediglich isolierter Merkmale eine distinktive Relevanz entwickeln. Das gilt, wenn auch nicht explizit ausformuliert, genauso für die akzeptierten Kategorien Affix oder Grundmorphem. Ein Affix ist typischerweise einsilbisch und unbetont mit wenig lautlichem Gehalt (ver-, be-, ge-, -er), aber es gibt Ausnahmen (hetero-, un-, -ei, -schaft). Ein Grundmorphem ist typischerweise bzw. präferiert frei, aber es gibt Ausnahmen (him-, psych(o)-). Die folgende Tabelle (Tab. 1) fasst die wichtigsten Merkmale der relevanten Einheiten zusammen. ${ }^{?}$

Um Missverständnisse zu vermeiden: Die Merkmale sind nicht als absolut, sondern als typisch zu verstehen, z.B. gibt es durchaus akzentuierbare Affixe wie ur-, -ier-. 


\begin{tabular}{|c|c|c|c|c|c|c|c|c|c|c|}
\hline Morphemtyp & Einheit & Beispiele & & & & cmale & & & & \\
\hline & & & 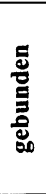 & 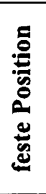 & 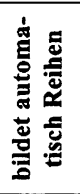 & 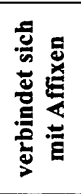 & 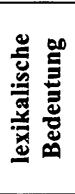 & 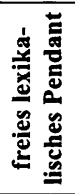 & 离 & 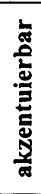 \\
\hline Affix & ge, ig, er & $\begin{array}{l}\text { Geäst, } \\
\text { glasig, } \\
\text { Kocher }\end{array}$ & + & + & + & - & - & - & - & - \\
\hline $\begin{array}{l}\text { Präfixoid, } \\
\text { Suffixoid }\end{array}$ & $\begin{array}{l}\text { riesen, } \\
\text { sau } \\
\text { werk, } \\
\text { los }\end{array}$ & $\begin{array}{l}\text { Riesentür, } \\
\text { Sauordmung, } \\
\text { Schuhwerk, } \\
\text { sorglos }\end{array}$ & + & + & + & - & - & + & + & - \\
\hline $\begin{array}{l}\text { freie lexikal. } \\
\text { Morpheme }\end{array}$ & $\begin{array}{l}\text { haus, } \\
\text { schloss, } \\
\text { stahl } \\
\end{array}$ & $\begin{array}{l}\text { Hausschuh, } \\
\text { Türschloss, } \\
\text { Stahlwerk }\end{array}$ & - & - & - & + & + & 0 & + & + \\
\hline
\end{tabular}

Tabelle 1: Zusammenfassung der Merkmale von Affixoiden

Mit Affixen haben Affixoide Gebundenheit, Positionsfestigkeit, automatische Reihenbildung, die Unfähigkeit, sich mit Affixen direkt zu verbinden und die fehlende lexikalische Bedeutung gemein. Das trennt sie damit von Kompositionsgliedern. Gleichzeitig heben sie sich von den Affixen durch das freie Pendant und tendenziell mehr Lautgehalt ab Affixoide bestehen nie aus einer einzigen schwahaltigen Silbe mit einfacher Silbenstruktur, genauso wenig wie Lexeme. Sie sind damit lautlich noch nicht reduziert wie viele der bereits etablierten Affixe (be- vs. ahd. $b \bar{l}$, ver-vs. ahd. faur, fra, fair, -er vs. lat. arius, vgl. Fleischer/Barz 1995) und stehen den Wörtern noch nahe. Das Kriterium des freien Pendants lässt sich auf Kompositionsglieder nicht anwenden. Präfixoide unterscheiden sich zudem von Gliedern im Determinativkompositum durch das Akzentmuster. Affixoide unterscheiden sich von Grundmorphemen semantisch, von Affixen lautlich und durch das freie Pendant.

Salopp gesprochen ist festzuhalten, dass sich Affixoide wie Affixe verhalten, aber aussehen wie Wörter.

Zwischen Prä- und Suffixoiden kommt es zu Unterschieden genauso, wie sich auch Präund Suffixe nicht ganz gleich verhalten. Suffixoidbildungen behalten das Akzentmuster des Determinativkompositums bei, Präfixoidbildungen verändern es. Außerdem sind die Produktivitätsgrade unterschiedlich. Beide entstehen gewöhnlich über anfänglich metaphorische Verwendung (vgl. bereits Tellenbach 1985). Die Präfixoidbildung ist äußerst vital, sie bevorzugt Spenderlexeme mit emotionalen Konnotationsmöglichkeiten (zur Semantik vgl. Leuschner i. Dr.). Suffixoide treten weniger häufig auf, da sich die Zweitglieder in Determinativkomposita als semantische Träger des Gesamtausdrucks sehr wahrscheinlich weniger leicht desemantisieren lassen als die Erstglieder, die als Zusatzinformation inhaltlich flexibler sein dürften und sich bei häufigerem Gebrauch leichter „abnutzen“. Damit wird der metaphorische Charakter der Zweitglieder, der zu einer Interpretation als Komposition führt, bewahrt und die inhaltliche Verselbstständigung der Einheit bleibt aus. 


\section{Warum der Begriff nötig ist}

Eine Nähe zu Metaphern existiert durchaus. Diese sollte aber entwicklungsbedingt verstanden werden insofern, als sich aus einer Metapher per Analogie ein oder zwei weitere bilden, die dann mit der Zeit die metaphorische Bedeutung einbüßen und Reihen bilden. Das heißt, die veränderte Bedeutung trägt das Affixoid selbst, sie entsteht nicht erst in der Verbindung mit einem Grundmorphem (vgl. Elsen 2004, S. 30). Neben den Kriterien Reihenbildung und freiem Pendant ist besonderes Augenmerk auf diesen semantischen Aspekt zu legen. Weil hier eindeutige Definitionen schwierig sind, kommt es zu Unterschieden bei der Einordnung vieler Einheiten, die es aber auch unter Verzicht auf die Kategorie Affixoid gibt. Darum wird im Folgenden ein Vorschlag für eine strengere Vorgehensweise gemacht, bei der dann einige Affixoidkandidaten ausscheiden. Ausgehend von den Beobachtungen in Barz (2006, S. 757), Decroos/Leuschner (2008) und Leuschner (i. Dr.), manche Beispiele hätten sowohl vergleichende als auch steigernde Bedeutung (stocksteif 'steif wie ein Stock', 'sehr steif'), werden folgende Kennzeichen für Affixoide angesetzt: Bei dem Affixoid handelt sich um eine produktiv verwendete, semantisch sich verselbstständigende Einheit, die parallel in ihrer ursprünglichen Bedeutung frei vorkommt. Einige Bildungen lassen sich jedoch bereits nicht mehr metaphorisch verwandt mit dem Einzellexem interpretieren.

So kann saublöd noch als 'blöd wie eine Sau' erklärt werden, nicht jedoch sauteuer. Affentheater mag als 'Theater, wie es ein Affe macht' gelten, aber eine solche Umschreibung passt nicht mehr zu Affenarbeit, Affenhitze. Laut Leuschner (i. Dr.) sind dabei zwei Entwicklungsschritte anzusetzen. Ähnliche Unterschiede werden bei Mordsangst oder Mordsgeschrei gegenüber Mordsding, Mordshunger, Mordshitze deutlich. Abgasfrei, bleifrei oder eisfrei sind mit 'frei von' zu umschreiben, aber rostfrei, bügelfrei, knitterfrei, scheinfrei nicht (ausführlich Vögeding 1981, Fandrych 1993), denn hier passt besser die Paraphrase mit 'ohne'. Herrenlos oder schuldenlos lassen sich mit 'gelöst, abgetrennt von' paraphrasieren, aber sorglos, furchtlos oder fehlerlos nicht (vgl. Fandrych 1993, S. 94, Fleischer/Barz 1995, S. 264, Simmler 1998, S. 442 f.), denn auch hier reicht 'ohne'. In manchen Fällen ist gar kein vernünftiger Zusammenhang mehr möglich (bombensicher, saugut, Mordsglück). Nicht die Frage, bei welchem Beispiel sich genau eine Grenze ziehen lässt, ist dabei relevant, sondern die Tatsache, dass sich das Affixoid bedeutungsmäßig verändert hat, gesehen für die Gruppe der Beispiele insgesamt. Bei Papst finden sich nämlich solche Verselbstständigungen nicht. Die Beispiele Skipapst, Literaturpapst, Kulturpapst und Kunstpapst (vs. Renaissancepapst, Gegenpapst) aus Muthmann (2001) sind Metaphern, die auf die erhöhte Sonderstellung eines Papstes anspielen. Auch in Formen mit luxus sind keine semantischen Verselbstständigungen zu verzeichnen, es bleibt stets bei der Umschreibung 'luxuriös, kostspielig (gesonnen), in den Bereich des Luxus gehörend', vgl. Luxusartikel, Luxusvilla, Luxusgeschöpf.

Nicht immer muss aber eine ursprüngliche bzw. metaphorische Beziehung erkennbar sein, denn es gibt auch Quereinsteiger, die bereits mit intensivierender Bedeutung und in Reihe verwendet werden ohne vorherige Reanalyse einzelner Komposita (vgl. Scheiß, Decroos/ Leuschner 2008, Leuschner i. Dr.). Damit gibt es nicht nur ein Modellwort (vgl. Motsch 1996, S. 167), sondern ein ganzes Modellmuster. Dieses von einzelnen metaphorischen Komposita unabhängige Verhalten bedeutet ein wichtiges Argument für die Annahme 
einer Wortbildungsart Affixoidbildung neben Derivation und Komposition. Hinzu kommt die unverkennbare Produktivität. Die Gruppe der sich ähnlich verhaltenden Einheiten ist groß und wächst an. Wir können momentan einen ,starken, noch nicht abgeschlossenen Ausbau dieses Funktionsstands in der neueren deutschen Sprachgeschichte" (Erben 1993, S. 85) beobachten. Einige Varietäten sind diesbezüglich besonders aktiv, vor allem die Jugendsprache (Unterhalterschiene, Liebesschiene, Funschiene, Tekkno-Zeugs, Amizeugs, flammwarm, flammneu, Killerband, Killerfolge, Killersound, vgl. Androutsopoulos (1998), endcool, endgeil, endstark, Klipptussi, Klippschule, Klipp-Job, oberdoll, Oberanturner, oberaffengeil, Oberklemmi, frustmäßig, spacemäßig, gorillamäßig, vgl. Elsen 2004). Die Präfixoidbildung ist besonders aktiv. Jugendliche benutzen das Muster, ein Lexem lediglich zur Verstärkung vor ein näher zu bestimmendes Wort zu setzen - eine aktive Wortbildungsmethode, die gerade auch im Alltag üblich ist. So treten in umgangssprachlichen, provokativ-kritisch belletristischen Werken Affixoidbildungen in bisher noch nicht untersuchter Breite und Intensität auf (z.B. arschfreundlich, saudoof, Riesenhallo, Riesenunterhaltung, Riesenspaß, Riesenauswahl, Riesenärger, scheiß-echt, scheißtoupiert, Scheiß-Farbe, Scheiß-Teleskop, faschomäßig, asimäßig, grundfreundlich, sündteuer, hochdramatisch, vollgültig, struntzbieder, strotzdumm, Schweinerock, Schweinegitarre, Hauptpreis, von Stuckrad-Barre, Doktor Oberlustig, Oberklemmi, Oberabgreifer, Obertempel, Oberhaifisch, karrieremäßig, mutterkomplexmäßig, satteltaschenmäßig, gesundheitstechnisch, aktualtechnisch, schwerreich, hochzivilisiert, Aids-profilaxe-technisch [sic!], Lindenberg). Das Bedürfnis nach emotional-emphatischer, ja aggressiver Ausdrucksweise, wie es in bestimmten Varietäten verstärkt vorkommt, steht in engem Zusammenhang mit einem relativ hohen Aufkommen an Präfixoidbildungen (vgl. über 5 $\%$ der Neologismen in der Jugendsprache gegenüber $0 \%$ in Fachsprachen, Elsen 2004) und unterstreicht die Funktionalität des Bildungsmusters.

Eine neue Wortbildungsart kann durchaus angesetzt werden. Aber da „seriöse“ Quellen für viele im Mittelpunkt stehen, fehlt das Belegmaterial, um die wahre Produktivität und Reichweite der Affixoidbildung zu fassen.

Insgesamt liegen also mehrere Gründe vor, Affixoide als Kategorie zu akzeptieren:

- Einige Argumente gegen die Kategorie gelten auch für die Zweierteilung, beispielsweise, dass es zu unklaren Einordnungen kommt. Andererseits wird gegen die Kategorie eingewandt, es gebe keinen für alle und nur für die betroffenen Elemente gültigen Kriterienkatalog. Den gibt es aber auch nicht für Affixe, wie Hansen/Hartmann (1991) zeigen. Ähnlich formuliert sollte es nur dann eine Kategorie geben, wenn sie sich durch eigene distinktive Merkmale von anderen Kategorien abhebt - das gilt jedoch auch nicht für Kompositionsglieder, denn nicht alle sind lexikalisch und frei (z.B. die Konfixe). Diese Argumente sprechen also nicht deutlich genug gegen eine Kategorie Affixoid.

- Die Trennlinie zwischen Komposition und Derivation ist nicht eindeutig. Es gibt aber nicht nur wenige Problemfälle, sondern eine ganze Gruppe von Wortbildungen, die weder klar dem einen noch dem anderen Typ zugeordnet werden kann und die sich systematisch ähnlich und relativ kohärent verhält. Der Bedarf nach einer dritten Kategorie neben Komposition und Derivation wird durch die bisher vorliegenden uneinheitlichen 
Analysen, konträren Entscheidungen, vor allem aber zusätzlichen Kategorievorschlägen bei gleichzeitiger Ablehnung des Begriffs deutlich.

- Die Gruppe dieser Einheiten mit systematischen morphosemantischen Gemeinsamkeiten wächst.

- Ursprüngliche Lexeme werden desemantisiert verwendet ohne metaphorische Grundlage und verselbstständigen sich. Es besteht keine Idiomatisierung einzelner komplexer Bildungen jeweils neu, sondern die Bedeutungsveränderung ist an eine Einheit wie riesen gekoppelt. Wer also Formen wie Riesenfreude, Riesenblamage, Riesenabenteuer immer einzeln als demotivierte Komposita bestimmt, vergisst dabei die Tatsache, dass neue Bildungen wie Riesenbildschirm nicht an sich die Bedeutung $\mathrm{zu}$ Riese verlieren, sondern aufgrund der anderen Bildungen, und dass riesen allein bereits demotiviert ist (vgl. auch Elsen 2004). Fandrych, der den Begriff des Affixoides ablehnt, formuliert die Beobachtung folgendermaßen: „Dabei gewinnen die Wortbildungsmuster mithilfe semantisch-funktionaler Analogiebildung sozusagen gerade bei größerer Produktivität - eine gewisse ,Eigendynamik'“ (Fandrych 1993, S. 108). Es hat sich also ein eigenes Modellmuster entwickelt.

- Ohne die Kategorie würden die Unterschiede in Beispielen wie bettelarm, bitterarm, wartungsarm und bügelarm nivelliert und höchstens durch zusätzlich Erklärungen und kontrastive Paraphrasen deutlich.

Darüber hinaus ist festzuhalten:

- Es treten vergleichbare Entwicklungen auch in anderen Sprachen auf (Bauer 1983, Ascoop 2005, Decroos/Leuschner 2008, Leuschner i. Dr.).

- Präfixoide schließen sehr oft als synthetische Elativformen eine morphologische Lücke im Deutschen, vgl. gro $\beta$, größer, riesengro $\beta$, am größten (Elsen 2004, S. 30). Der Versprachlichungsbedarf von emotional stark markierten Inhalten wird offenbar mit dem im Deutschen zu Verfügung stehenden Morphemrepertoire nicht gedeckt. Dieser Aspekt ist vor allem auch im Fremdsprachenunterricht mit der entsprechenden Terminologie leichter zu fassen.

- Affixoide müssen sich nicht unbedingt in Richtung Affixe entwickeln. Sie können auch ihren Status beibehalten. Es geht nicht notwendigerweise um Elemente, die sich auf dem Weg zum Derivationsaffix befinden, das lässt sich synchron gar nicht feststellen, sondern um Wörter, die die Funktion von Affixen übernehmen, die in dieser Hinsicht also keine Wörter sind, da sie sich von ihnen unterscheiden, Affixe aber auch nicht. Gerade dann aber brauchen wir einen Begriff.

\section{Eine kognitiv-grammatische Sichtweise}

Präfixoidbildungen wie Riesenhunger oder Affenhitze sind ein Muster, das ausgeprägtes stilistisches Potenzial besitzt, zudem aber auch eine lexikalisch-morphologische Lücke schließt, sich verselbstständigt und ausdehnt. Solch eine gebrauchs- und funktionsabhängige Dynamik spielt in verschiedenen Ansätzen eine Rolle, u.a. auch in der Kognitiven Grammatik. Sie verbindet Einsichten, die aus der Prototypentheorie bekannt sind, mit 
Erkenntnissen des Konnektionismus bzw. Netzwerkgedankens. Aber auch einige Ideen der Grammatikalisierung finden sich hier wieder (Elsen 1999).

Folgende Grundannahmen sind charakteristisch (Langacker 1987, 1991, Taylor 2002, Croft/Cruse 2007): Sprachwissen ist dynamisch. Die Grammatik ist gebrauchs- und oberflächenorientiert. Komplexe Strukturen lassen sich aus vielen Einzelbeispielen abstrahieren; durch häufigen Gebrauch verfestigt bzw. verselbstständigt sich eine Struktur und kann schließlich den Status einer Einheit erhalten und dieser kann nur graduell, nicht diskret, verstanden sein. Wissen kann verblassen. Syntaktische und morphologische Strukturen unterliegen den gleichen Operationen. Zwischen Lexikon und Syntax/Morphologie gibt es keine eindeutigen Grenzen.

Weil die Sprache nicht unabhängig von den Sprechern und Sprechsituationen existiert, ist ein Zusammenspiel von Sprache und anderen Kenntnisbereichen erlaubt. Neuronale Tatsachen stehen in Verbindung mit Sprachstrukturen. Neuropsychologische, biologische und psychologische Fakten bilden eine sprachexterne Basis für neue Erklärungsansätze von Sprechen, Spracherwerb und Sprachwandel. Dadurch ändert sich auch der bisher mathematisch orientierte Regelbegriff. Die gesamte aristotelisch begründete Vorstellung von diskreten Kategorien, die sich durch eine Gruppe von Merkmalen charakterisieren lassen, über die alle Mitglieder in gleicher Weise verfügen, wird aufgegeben. Stattdessen können Eigenschaften mehr oder weniger treffend bzw. gut sein. Kriterienbündel dürfen statt lediglich isolierter Merkmale eine distinktive Relevanz entwickeln.

In ihrer Arbeit zu aktuellen Bildungen auf -frei, -arm, -voll etc. plädieren Brdar-Szabó/ Brdar (2000) gegen eine dritte Kategorie zwischen Derivation und Komposition und für eine Übergangszone, weil das sonst im Endeffekt zur Annahme von mehr und mehr Prototypen und immer mehr und kleineren Kategorien führe, ein ,infinitesimalization of the phenomenon" (Brdar-Szabó/Brdar 2000, S. 147). Bei dieser Argumentation werden aber charakteristische Prototypeneffekte, die Anziehungskraft eines offenbar guten Beispiels auf mehr und mehr ähnliche Wörter und damit Verstärkung und Verselbstständigung des Musters, übersehen. Denn die Verteilung der Beispiele zwischen dem Kompositum-Prototypen und dem Derivationsprototypen ist nicht gleichmäßig, vielmehr siedeln sich um Formen à la Riesenhunger immer mehr Bildungen erstens mit riesen in dieser speziellen Funktion und zweitens weiterer solcher Einheiten in vergleichbarer Bedeutung an. Dies zeigen die o.g. Untersuchungen verschiedener umgangssprachlich orientierter Varietäten. Das Schema Präfixoidbildung zieht mittlerweile auch neue Formen an, die nicht, wie ursprünglich zumeist, auf einem metaphorisch verwendeten Kompositum beruhen, sondern gleich mit eigener Bedeutung des ersten Glieds gebildet werden (endcool, endstark bzw. endgeil). Die Einheiten tragen ihre neue Bedeutung in Abhängigkeit von dieser Bildungsweise. Dies weist auf die Selbstständigkeit des Musters hin. Seine starke Anziehungskraft dürfte im Wesentlichen durch das stilistische Potenzial, sehr emphatisch, ja drastisch Dinge besonders gut oder schlecht erscheinen zu lassen, bedingt sein. Gerade die Präfixoidbildungen schließen, wie bereits erwähnt, als synthetische Elativformen eine morphologische Lücke im Deutschen. So kommt es zu einem Cluster, das die Annahme einer eigenen Kategorie durchaus verdient. Dies schließt ganz im Sinne der Kognitiven Grammatik Übergangszonen und schwer einordbare Beispiele nicht aus. Sie sind aber erstens im Falle der Präfixoidbildungen nicht so häufig im Vergleich zu den typischen 
Beispielen. Zweitens kann sich ein Problembeispiel bei genügend Nachahmern vom Prototypen weiter anziehen lassen. Die Sprecher entscheiden selbst, ob es bei wenig metaphorisch begründbaren Komposita Literaturpapst und Modepapst bleibt oder ob das Muster ausgeweitet wird.

Im Laufe der Diskussion sollte deutlich geworden sein, dass mehr und mehr Bedarf besteht, die beschriebene Gruppe an Einheiten kognitiv und begrifflich zu fassen. Aber offenbar fehlt häufig noch das Verständnis aktueller Vorgänge, denn die tatsächliche Verbreitung solcher Bildungen ist noch gar nicht erkannt. Der Begriff Affixoid ist nicht bloße intellektuelle Spielerei und es geht auch längst nicht mehr um gute oder schlechte Komposita bzw. Ableitungen wie seit Beginn der Diskussion, sondern um ständig neue Bildungen mit eigener Dynamik. Natürlich besteht grundsätzlich kein Problem, ob zwischen Türmen mit und ohne Spitze, groß oder klein, unterschieden wird oder nicht (engl. turret, tower, pinnacle, steeple, dt. Turm). Es gibt auch ohne einen Begriff die kognitive Wahrnehmung. Aber Begriff und Kategorie gehen einher, sie helfen der Verständigung und dem Bewusstmachen von Gemeinsamkeiten und Unterschieden - denn nur was benannt wird, kann auch verstanden werden.

\section{Literatur}

Altmann, Hans/Kemmerling, Silke (2000): Wortbildung fürs Examen. Wiesbaden.

Androutsopoulos, Jannis K. (1998) : Deutsche Jugendsprache. Untersuchungen zu ihren Strukturen und Funktionen. Frankfurt/M. et al.

Ascoop, Kristin (2005): Affixoidhungrig? Skitbra! Status und Gebrauch von Affixoiden im Deutschen und Schwedischen. In: Germanistische Mitteilungen 62, S. 17-28.

Barz, Irmhild (2006): Die Wortbildung. In: Duden. Die Grammatik. Mannheim et al. S. 641-772.

Bauer, Laurie (1983): English Word-Formation. Cambridge et al.

Bergmann, Rolf/Pauly, Peter/Stricker, Stefanie (2001): Einführung in die deutsche Sprachwissenschaft. 3. Aufl. Heidelberg.

Brdar-Szabó, Rita/Brdar, Mario (2000): Grammaticalization and the lexicon: Core-and-periphery model vs prototype approach. In: Mangasser-Wahl, Martina: Prototypentheorie in der Linguistik. Tübingen. S. 139-159.

Croft, William/Cruse, D. Alan (2007): Cognitive Linguistics. 4. Aufl. Cambridge.

Decroos, Nancy/Leuschner, Torsten (2008): Wortbildung zwischen System und Norm: Affixoide im Deutschen und im Niederländischen. In: Sprachwissenschaft 33, S. 1-34.

Donalies, Elke (2002): Die Wortbildung des Deutschen. Ein Überblick. Tübingen.

Eichinger, Ludwig M. (2000): Deutsche Wortbildung. Eine Einführung. Tübingen.

Eisenberg, Peter (2006): Grundriß der deutschen Grammatik. Band 1. Das Wort. 3. Auflage. Stuttgart/ Weimar.

Elsen, Hilke (1999): Ansätze zu einer funktionalistisch-kognitiven Grammatik. Konsequenzen aus Regularitäten des Erstspracherwerbs. Tübingen.

Elsen, Hilke (2004): Neologismen. Formen und Funktionen neuer Wörter in verschiedenen Varietäten des Deutschen. Tübingen.

Elsen, Hilke (2005a): Das Kunstwort. In: Muttersprache 115.2, S. 142-149.

Elsen, Hilke (2005b): Deutsche Konfixe. In: Deutsche Sprache 33, S. 133-140. 
Elsen, Hilke (eingereicht): Between phonology and morphology. In: Michel, Sascha/Onysko, Alexander: Cognitive Approaches to Word Formation. Berlin/New York.

Elsen, Hilke/Michel, Sascha (2007): Wortbildung und Sprachgebrauch. Desiderate und Perspektiven einer etablierten Forschungsrichtung. In: Muttersprache 1/2007, S. 1-16.

Engel, Ulrich (1988): Deutsche Grammatik. 2. Aufl. Heidelberg.

Erben, Johannes (1993): Einführung in die deutsche Wortbildungslehre. 3. Aufl. Berlin.

Fandrych, Christian (1993): Wortart, Wortbildung und kommunikative Funktion. Am Beispiel der adjektivischen Privativ- und Possessivbildungen im heutigen Deutsch. Tübingen.

Fleischer, Wolfgang (1982): Wortbildung der deutschen Gegenwartssprache. 5. Aufl. Tübingen.

Hansen, Sabine/Hartmann, Peter (1991): Zur Abgrenzung von Komposition und Derivation. Trier.

Henzen, Walter (1957): Deutsche Wortbildung. 2. Aufl. Tübingen.

Kluge, Friedrich (1999): Etymologisches Wörterbuch der deutschen Sprache. Bearbeitet von Elmar Seebold. 23. Aufl. Berlin/New York.

Krahe, Hans, Meid, Wolfgang (1967): Germanische Sprachwissenschaft III. Wortbildungslehre. Berlin.

Lachachi, Djamel Eddine (2008): Zur Stellung der Halbaffigierung in der deutschen Wortbildung. In: Eichinger, Ludwig M./Meliss, Meike/Dominguez Vázquez, María J.: Wortbildung heute. Tendenzen und Kontraste in der deutschen Gegenwartssprache. Tübingen. S. 213-229.

Langacker, Ronald W. (1987): Foundations of Cognitive Grammar. Volume I. Theoretical Prerequisites. Stanford.

Langacker, Ronald W. (1991): Foundations of Cognitive Grammar. Volume II. Descriptive Application. Stanford.

Langacker, Ronald W. (2000): Grammar and Conceptualization. Berlin/New York.

Leuschner, Torsten (im Druck): Ausnahmepianist fettgeschreckt - inbleich! Deutsche, niederländische und schwedische Präfixoide im Spannungsfeld von Genealogie, Kreativität und Norm. In Zusammenarbeit mit Kristin Ascoop und Nancy Decroos. In: Germanistische Linguistik.

Lohde, Michael (2006): Wortbildung des modernen Deutschen. Ein Lehr- und Übungsbuch. Tübingen.

Lühr, Rosemarie (1996): Neuhochdeutsch. 5. Aufl. München.

Motsch, Wolfgang (1996): Affixoide. Sammelbezeichnung für Wortbildungsphänomene oder linguistische Kategorie? In: Deutsch als Fremdsprache 33, S. 160-168.

Motsch, Wolfgang (1999): Deutsche Wortbildung in Grundzügen. Berlin/New York.

Muthmann, Gustav (2001): Rückläufiges Wörterbuch. Handbuch der Wortausgänge im Deutschen, mit Beachtung der Wort- und Lautstruktur. 3. Aufl. Tübingen.

Naumann, Bernd (1972): Wortbildung in der deutschen Gegenwartssprache. Tübingen.

Olsen, Susan (1986): „Argument-Linking“ und unproduktive Reihen bei deutschen Adjektivkomposita. In: Zeitschrift für Sprachwissenschaft 5.1, S. 5-24.

Paul, Hermann (1937): Prinzipien der Sprachgeschichte. 5. Aufl. Halle.

Petermann, Heinrich (1971): Semantische Veränderungen erster Kompositionsglieder im Grenzbereich zwischen Zusammensetzungen und Präfixbildungen. In: Deutsch als Fremdsprache 8, S. 108-113.

Schmidt, Günter Dietrich (1987a): Das Kombinem. Vorschläge zur Erweiterung des Begriffsfeldes und der Terminologie für den Bereich der Lehnwortbildung. In: Hoppe, Gabriele/Kirkness, Alan/Link, Elisabeth/Nortmeyer, Isolde/Rettig, Wolfgang/Schmidt, Günter Dietrich: Deutsche Lehnwortbildung. Tübingen. S. 37-52. 
Schmidt, Günter Dietrich (1987b): Das Affixoid. Zur Notwendigkeit und Brauchbarkeit eines beliebten Zwischenbegriffs der Wortbildung. In: Hoppe, Gabriele/Kirkness, Alan/Link, Elisabeth/Nortmeyer, Isolde/Rettig, Wolfgang/Schmidt, Günter Dietrich: Deutsche Lehnwortbildung. Tübingen. S. 53-101.

Simmler, Franz (1998): Morphologie des Deutschen. Flexions- und Wortbildungsmorphologie. Berlin.

Stevens, Christopher M. (2005): Revisiting the Affixoid Debate: On the Grammaticalization of the Word. In: Leuschner, Torsten/Mortelmans, Tanja/De Groodt, Sarah: Grammatikalisierung im Deutschen. Berlin/ New York. S. 71-84.

Taylor, John R. (2002): Cognitive Grammar. Oxford.

Tellenbach, Elke (1985): Wortbildungsmittel im Wörterbuch. Zum Status der Affixoide. In: Linguistische Studien / ZISW A 122. Berlin. S. 266-315.

Vögeding, Joachim (1981): Das Halbsuffix „-frei“. Zur Theorie der Wortbildung. Tübingen.

Wilmanns, Wilhelm (1899): Deutsche Grammatik. Gotisch, Alt-, Mittel- und Neuhochdeutsch. Zweite Abteilung: Wortbildung. 2. Aufl. Straßburg.

Wilss, Wolfram (1986): Wortbildungstendenzen in der deutschen Gegenwartssprache. Tübingen.

\section{Belegquellen}

Lindenberg, Udo (1989): El Panico. München.

Von Stuckrad-Barre, Benjamin (1998): Soloalbum. Hamburg.

Prof. Dr. Hilke Elsen

Institut für Deutsche Philologie

Ludwig-Maximilians-Universität München

Schellingstr. 3

D-80799 München

E-Mail: hilkee@Irz.uni-muenchen.de 